\title{
Medical Toxicology and Public Health-Update on Research and Activities at the Centers for Disease Control and Prevention, and the Agency for Toxic Substances and Disease Registry: Introduction to the Laboratory Response Network-Chemical (LRN-C)
}

\author{
Carl Skinner, $M D^{1-4}$, Jerry Thomas, $M D^{1-4}$, Rudolph Johnson, $P h D^{1}$, Robert Kobelski, $P h D^{1}$
}

\author{
${ }^{1}$ Division of Laboratory Sciences, National Center for Environmental Health (NCEH), Atlanta, GA \\ ${ }^{2}$ Department of Emergency Medicine, Section of Toxicology, Emory University School of Medicine, Atlanta, GA \\ ${ }^{3}$ Emory/CDC Medical Toxicology Fellowship, Atlanta, GA \\ ${ }^{4}$ Georgia Poison Control Center, Atlanta, GA
}

\begin{abstract}
The following is an update on activities in which medical toxicologists are actively involved at CDC/ATSDR. The Journal of Medical Toxicology periodically will highlight some of these activities to illustrate the growing relationship between clinical toxicology and public health.
\end{abstract}

\section{LABORATORY RESPONSE NETWORK- CHEMICAL (LRN-C)}

Planning and preparing for a mass chemical, biological, or radiological exposure remains challenging. An effective response to such an event involves coordinating resources on the federal, state, and local levels as well as participation of local healthcare facilities and providers who will struggle to maintain surge capacity above and beyond existing demands. As a result of responses to the anthrax attacks in 2001, many clinicians became familiar with the Laboratory Response Network (LRN) and the public health laboratory infrastructure in place to deal with biological threats. In contrast, many clinicians and toxicologists are less aware of the existence and capabilities of the chemical capabilities of the LRN (LRN-C), which was developed to respond to a mass chemical exposure (MCE).

There are 3.1 billion tons of hazardous materials shipped annually in the United States. Of these, there are $>800,000$ shipments daily of toxic industrial chemicals (TICs) or toxic industrial materials (TIMs) [1]. MCE events may be overt and easily recognized, or covert with more subtle effects. A chemical event-intentional or accidental—may have serious physical as well as psychological consequences to the public. Emergency departments (EDs) may be inundated with real and potential victims. Emergency medicine (EM) physicians, poison control centers (PCCs), and toxicologists will be on the frontline during a chemical event and will play a vital role in coordinating the activities of the LRN-C with other public health resources.

Past incidents highlight the potential for accidental or intentional exposure of populations to hazardous materials. In Japan in 1994, Aum Shinrikyo, a religious cult, used sarin in Matsumoto City to kill 7 people and cause $>600$ people to be exposed to the chemical [2]. Nine months later, they released sarin into the subways of Tokyo, resulting in 9 deaths, 1,200 exposures, and 5,500 patient evaluations. In Graniteville, SC in 2005, a train transporting chlorine gas collided with a parked train [3]. The accident caused a breach in one of the transporting tanker cars, releasing $>11,000$ gallons of chlorine gas into the community. This accident resulted in 9 deaths and $>500$ people seeking medical attention. These events illustrate the vast number of patients, including the critically ill, moderately symptomatic, and those worried about being potentially exposed, who need medical care following an MCE.

Note: The findings and conclusions in this article are those of the authors and do not necessarily represent the views of the Centers for Disease Control and Prevention (CDC) or the Agency for Toxic Substances and Disease Registry (ATSDR). 
Physicians should anticipate and plan for the chaos of MCEs. During the initial hours of an event, healthcare workers will be faced with incomplete or incorrect information as well as poor communication. Initial treatment will be empirical and based on incomplete exposure information. Emergency response personnel, EM physicians, toxicologists, and PCCs will be at the forefront of the public health response. Therefore it will be imperative to know how to alert and integrate with the public health infrastructure early to enable mobilization of appropriate resources that may aid in patient care.

Local authorities such as fire departments and hazardous materials teams (HAZMAT) have environmental field-testing equipment and methods that rely on varying technologies, but these may yield unreliable results. Currently, the equipment being used for field-testing is not adequately tested in real settings and standards have not been set for its use. This is significant because inaccurate results may lead to inappropriate treatment or improper utilization of resources. Further, most hospital laboratories and reference laboratories will not have methods capable of confirming chemical exposures. The experience of many toxicologists will be that patient management will be empirical and based on presentation, not on specific laboratory results. Analysis of clinical samples may aide in identifying the agent and coordinating treatment for those severely exposed and help to reassure those not exposed. Testing may also identify those who merit long-term medical follow-up for adverse health effects that can result from low-level exposures.

Through a collaborative effort involving the Centers for Disease Control and Prevention (CDC), the Federal Bureau of Investigation (FBI) and the Association of Public Health Laboratories, the LRN started operating August 1999. The LRN-C consists of 62 state, territorial, and metropolitan public health laboratories. Its objective is to ensure an effective laboratory response to biological and chemical terrorism events by helping to improve the nation's public health laboratory infrastructure (http://www.bt.cdc.gov/lrn/ factsheet.asp).

The LRN has been involved in testing for infectious diseases such as avian influenza and SARS, and carries out surveillance with the Biowatch program. The LRN-C has developed blood and urine assays for specific chemical agents of concern in a public health event. Other federal laboratories (FBI, US Environmental Protection Agency, US Food and Drug Administration, and US Department of Agriculture) may be involved in an event for testing chemical agents in food, water, and environment. The LRN-C is primarily tasked with providing analytical data of clinical samples from patients in order to guide public health response.

LRN-C laboratories are designated as level 1, 2, or 3 based on their capabilities. A level 3 laboratory works with local hospitals and laboratories for training on correctly collecting and correctly transporting specimens, coordinating with PCCs as sentinel indicators of chemical exposure, and issuing chain of custody. Level 2 laboratories have chemists who are trained to test clinical samples for biomarkers of certain agents such as cyanide, nerve agents, and toxic metals. The capabilities of level 1 laboratories extend beyond those of the level two laboratories to include testing for biomarkers of additional chemical warfare agents and naturally occurring toxins. The LRN-C laboratories also provide additional laboratory capacity to supplement CDC. CDC's laboratory serves as the central reference laboratory, providing training and proficiency testing to other LRN-C members.

The 2 primary goals of the LRN-C are to produce interpretable laboratory results on a limited number of clinical samples in a timely manner and to have flexible laboratory capabilities that can be utilized for extended capacity during chemical terrorism events. When large numbers of samples must be tested, level 1 laboratories can be used as surge capacity to analyze thousands of clinical samples. The results of these tests would then be sent to $\mathrm{CDC}$, where they are combined, interpreted, and the data reported to the requesting party.

To ensure quality in its methods and reporting, CDC continually provides training to members of the LRN-C. An initial laboratory validation and continuing proficiency testing ensures that the highest standards are maintained. Through regular proficiency testing and education, each laboratory in the LRN-C is ready and capable to respond to a chemical event.

LRN-C methods have been applied to identify agents and persons who were or were not exposed in incidents of suspected chemical agent exposures. In one incident, a military serviceman was exposed to a chemical warfare agent following an attempt to render safe an unexploded World War I munition that had been dredged up and found in a residential driveway. He received chemical burns and blisters to his upper extremities and spent 2 weeks in the hospital. Using new methods, the LRN-C was able to detect sulfur mustard adducts from his blood, blister fluid, and metabolites in his urine. While small-scale events such as this have demonstrated the utility of the analytical method capabilities of the LRN-C, regular participation in large-scale exercises ensures readiness for larger events. These real-time exercises involve all aspects of response, including initial communications and approval, collection team deployment, sample transportation, sample analysis, quality control approval, data reporting, and surge capacity.

CDC recently participated in an expanded capacity exercise in which 5,000 samples were tested at CDC laboratories and an additional 5,000 samples were tested by the level 1 LRN-C laboratories. This exercise was intended to simulate the challenges encountered during a large-scale event in which sample analysis capacity would be pushed to its limits.

The response to any significant public health threat will involve multiple groups including clinicians and public health and law enforcement agencies. Early and consistent communication among agencies is important. Following the Tokyo sarin attacks, for example, the police knew which agents had been used long before hospitals knew of them $[2,4]$. A breakdown in communication after the attack made it difficult for physicians to treat patients who were experiencing symptoms from an unknown substance. It is therefore important for all physicians, medical toxicologists in particular, to be familiar with local and state public 


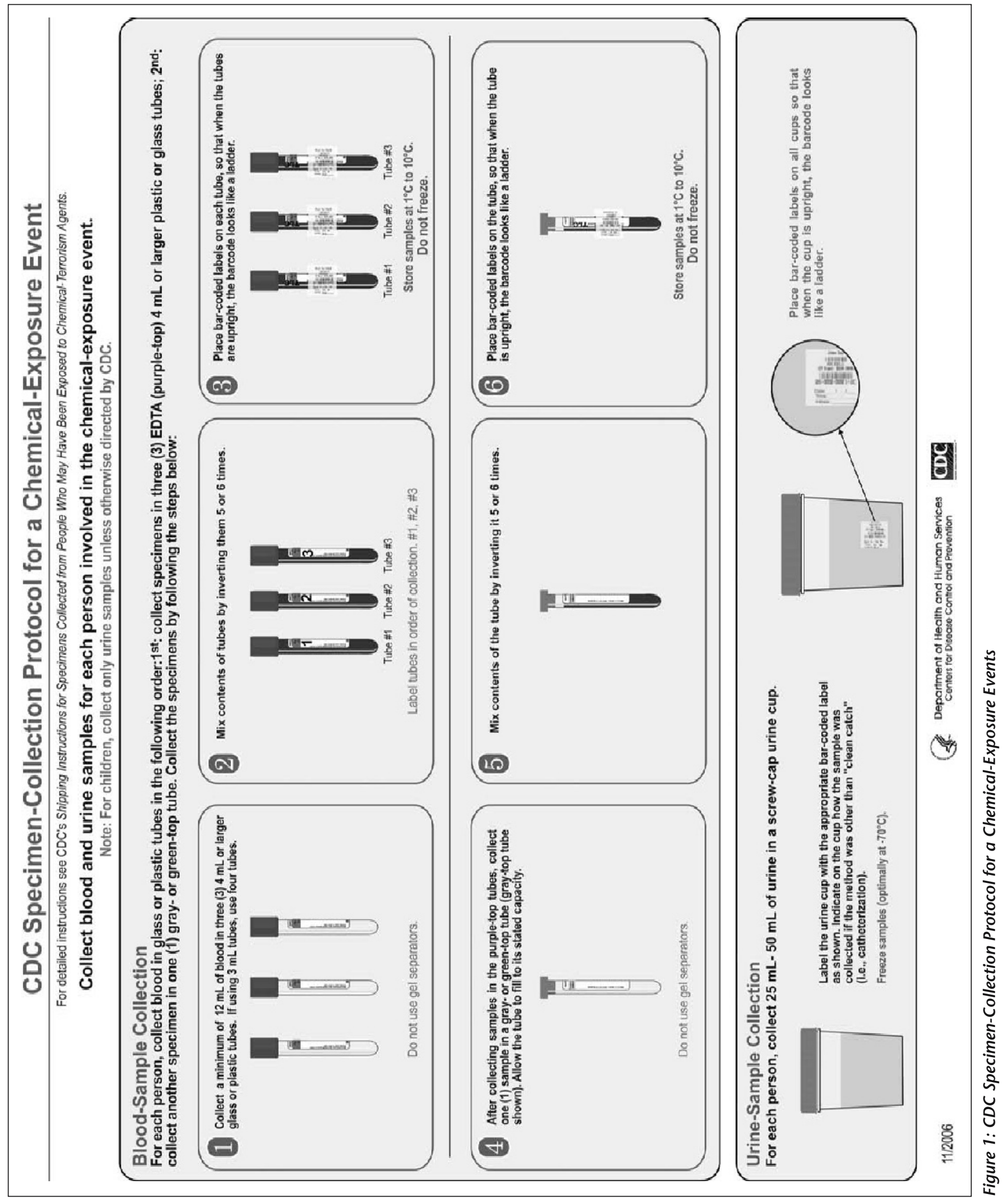


health agencies and to possess current contact information. These relationships are best established in advance, well before a crisis occurs. During an event response, clinical samples such as blood and urine most likely will need to be collected from local hospitals. Blood testing methods include those for cyanide, metals, and volatile organic compounds. However, many assays have been developed to identify metabolites in urine. Therefore during an event response, urine should be collected and frozen if analysis is anticipated. Collecting and shipping procedures for suspected or known chemical-associated specimens can be found on CDC's website at http://www.bt.cdc.gov/chemical/lab.asp (Figure 1).

\section{SUMMARY}

Medical toxicologists and PCCs should understand the operation of local, state, and federal public health infrastructure; the capabilities of laboratories used; and recognize their own roles as a vital component of public health response. Phone numbers for local health departments should be readily available in EDs and PCCs. PCC staff should also be aware of the LRN-C and its sampling and shipping procedures, as they might be contacted before public health officials or locally based medical toxicologists for guidance. CDC staff, including medical toxicologists, provides multiple educational activities and programs to improve the knowledge of EMs regarding the capabilities of the LRN-C.

\section{REFERENCES}

1. US Department of Transportation Pipeline and Hazardous Materials Safety Administration [webpage on the Internet]. Hazardous materials shipments [updated 2005; cited 2007 Nov]. Available from: http://hazmat.dot.gov/pubs/hms/ hmship.htm

2. Tokuda Y, Kikuchi M, Takahashi O, Stein GH. Prehospital management of sarin nerve gas terrorism in urban settings: 10 years of progress after the Tokyo subway sarin attack. Resuscitation 2006 Feb;68(2):93-202.

3. Centers for Disease Control and Prevention (CDC). Public health consequences from hazardous substances acutely released during rail transit-South Carolina, 2005; selected States, 1999-2004. MMWR Morb Mortal Wkly Rep 2005 Jan; 28; 54(3):64-67.

4. Okumura T, Suzuki K, Fukuda A, et al. The Tokyo subway sarin stack: Disaster management. Part 2: Hospital response. Acad Emerg Med 1998;5(6):618-624. 